\title{
Creating semiclassical black holes in collider experiments and keeping them on a string
}

\author{
G. Dvali ${ }^{a b}$ and S. Sibiryakov ${ }^{a c}$ \\ a Theory Group, Physics Department, CERN, \\ CH-1211 Geneva 23, Switzerland \\ ${ }^{b}$ Center for Cosmology and Particle Physics, \\ Department of Physics, New York University, \\ 4 Washington Place, New York NY 10003, U.S.A. \\ ${ }^{c}$ Institute for Nuclear Research of the Russian Academy of Sciences, \\ 60th October Anniversary prospect, 7a, 117312 Moscow, Russia \\ E-mail: Georgi.Dvali@cern.ch, sibir@ms2.inr.ac.ru
}

ABSTRACT: We argue that a simple modification of the $\mathrm{TeV}$ scale quantum gravity scenario allows production of semiclassical black holes in particle collisions at the LHC. The key idea is that in models with large extra dimensions the strength of gravity in the bulk can be higher than on the brane where we live. A well-known example of this situation is the case of warped extra dimensions. Even if the energy of the collision is not sufficient to create a black hole on the brane, it may be enough to produce a particle which accelerates into the bulk up to trans-Planckian energy and creates a large black hole there. In a concrete model we consider, the black hole is formed in a collision of the particle with its own image at an orbifold plane. When the particle in question carries some Standard Model gauge charges the created black hole gets attached to our brane by a string of the gauge flux. For a 4-dimensional observer such system looks as a long-lived charged state with the mass continuously decreasing due to Hawking evaporation of the black hole. This provides a distinctive signature of black hole formation in our scenario.

KEYWORDs: Large Extra Dimensions, Black Holes. 


\section{Contents}

1. Introduction 1

2. Toy model 田

3. String of gauge flux 7

4. Generalizations and summary 12

A. Stability of the string in warped background 13

B. Internal oscillations of BH-string bound system 15

\section{Introduction}

Searches of the physics beyond the Standard Model (SM) at the Large Hadron Collider (LHC) are predominantly motivated by the Hierarchy Problem, the inexplicable stability of the weak scale versus the Planck mass. One approach to this issue [1] is that the hierarchy is maintained by a low, around a few $\mathrm{TeV}$, value of the quantum gravity scale $M_{*}$ which is a natural cutoff of the theory. Originally, this idea was proposed in the context of theories with extra dimensions. In order to account for the weakness of the gravitational interaction at large distances the size of extra dimensions in this approach has to be relatively large. The SM fields are then assumed to be localized on the 4-dimensional worldvolume of a 3-brane embedded into the higher-dimensional bulk. It is worth mentioning that, as understood recently [2, 3], the class of theories with low scale of quantum gravity is actually wider and includes generic theories with large number of particle species.

The common collider signature of theories with low quantum gravity scale would be production of mini black holes (BHs) once the energy of particle collision exceeds $M_{*}$ (see, e.g., the second reference in [1]). On the other hand the theoretically best understood properties, such as the Beckenstein entropy [4] and Hawking radiation [5], are related to semiclassical BHs with large masses, $M_{\mathrm{bh}} \gg M_{*}$. It is important to know the chances of observing such BHs at the LHC. This subject was discussed in refs. [6-10].

The standard approach [6, 0, 9, 10] (see [11] for a recent review) to $\mathrm{BH}$ production at colliders assumes that BHs are formed directly in the collision of two SM particles. Then the most experimentally accessible BHs are the light ones with the mass $M_{\mathrm{bh}}$ near the quantum gravity scale $M_{*} \sim \mathrm{TeV}$. However, the production rates and signatures of such BHs are clouded by theoretical uncertainties. In contrast to large BHs with $M_{\mathrm{bh}} \gg M_{*}$ which evaporate into a high multiplicity thermal final state via Hawking radiation, the 
light BHs are likely to decay into only a few high energy particles: a signal difficult to disentangle from the background. Besides, there are several effects which can push the collision energy needed for BH formation considerably higher than $M_{*}$ [12]. These include the energy loss by colliding particles prior to the formation of the $\mathrm{BH}$ horizon and the effects of the charge [13]. Within the standard scenario this makes observation of semiclassical BHs at the LHC problematic even in the most optimistic case $M_{*} \sim 1 \mathrm{TeV}$ and virtually impossible if $M_{*} \gtrsim 10 \mathrm{TeV}$.

In this paper we take another approach. Generically, theories with large extra dimensions contain particles which can freely propagate in the bulk. These particles can be produced in collider experiments once the extra dimensions open up. On the other hand, the scale of quantum gravity may depend on the position in extra dimensions. In particular, this scale can be much lower in the bulk than on the brane. Thus, one may imagine sending particles from the brane to the region of strong gravity and producing a large (much larger than the Planck length at that point) semiclassical BH there. This possibility was first pointed out in ref. [8].

A particular class of theories where the strength of gravitational interaction grows into the bulk is provided by models with warped compactification. We emphasize that we consider the class of models where the parameter $M_{*}$ entering into the gravitational action

$$
S_{g}=-M_{*}^{d-2} \int d^{d} x \sqrt{|g|} R
$$

is constant over the whole space-time. In the expression (1.1) $d$ is the total number of dimensions and $R$ is the Ricci scalar. The parameter $M_{*}$ sets the strength of gravitational interactions from the point of view of a local bulk observer and so this strength is constant. However, the situation is different from the point of view of a 4-dimensional observer who can make measurements only on the brane. Let us clarify this issue.

Consider a general higher-dimensional metric invariant under the 4-dimensional Poincare group,

$$
d s^{2}=A^{2}(y) \eta_{\mu \nu} d x^{\mu} d x^{\nu}+\gamma_{i j}(y) d y^{i} d y^{j}
$$

where $\eta_{\mu \nu}$ is the 4-dimensional Minkowski metric, and the coordinates $y^{i}$ represent extra dimensions. The SM brane is located at some point $y=y_{b}$; we set $A\left(y_{b}\right)=1$. Generically, the warp factor $A^{2}(y)$ in front of the 4-dimensional metric depends on the position in extra dimensions. In particular, it can be smaller in the bulk than on the brane, $A(y)<1$ for some $y$. Imagine now that two particles are sent from the brane into the bulk along parallel trajectories with separation $\Delta x$ between them. Let us neglect for the moment gravitational attraction between the particles. Then, the coordinate distance $\Delta x$, which characterizes the separation between the particles for the brane observer, stays constant while the distance measured in the local reference frame of the bulk observer decreases as

$$
r=A(y) \Delta x
$$

A similar effect occurs for the energies of the particles. While for the brane observer the energies of the particles remain constant and equal to their initial values $\mathcal{E}^{(1)}, \mathcal{E}^{(2)}$, for the 
bulk observer the energies are blue-shifted due to the warp factor,

$$
E^{(i)}=\mathcal{E}^{(i)} / A(y)
$$

Consider now gravitational interaction between the particles. The strength of this interaction is characterized by the dimensionless ratio of the potential gravitational energy $U$ to the total energy of the system. In the linear approximation the bulk observer estimates this ratio as,

$$
\frac{-U}{E^{(1)}+E^{(2)}} \sim \frac{E^{(1)} E^{(2)}}{M_{*}^{d-2} r^{d-3}\left(E^{(1)}+E^{(2)}\right)} .
$$

When expressed in terms of the quantities relevant for the brane observer the ratio takes the form,

$$
\frac{-U}{E^{(1)}+E^{(2)}} \sim \frac{\mathcal{E}^{(1)} \mathcal{E}^{(2)}}{\left[A(y) M_{*}\right]^{d-2} \Delta x^{d-3}\left(\mathcal{E}^{(1)}+\mathcal{E}^{(2)}\right)} .
$$

We see that the strength of the gravitational interaction of particles at point $y$ in the bulk, when viewed from the brane, is governed by an effective Planck mass

$$
M_{*}^{\mathrm{eff}}=A(y) M_{*} .
$$

According to our assumption about the function $A(y)$ this effective Planck mass decreases from the brane into the bulk. In this sense we say that gravity in models with warped geometry is stronger in the bulk than on the brane.

Clearly, the process consisting of simultaneous production of two bulk particles and their subsequent collision in the strong gravity region to create a $\mathrm{BH}$ is highly improbable. At the same time, from the bulk point of view it is clear that a single particle cannot produce a $\mathrm{BH}$ unless there is some mechanism to convert its kinetic energy into a centerof-mass energy of a collision. Luckily, there is a natural candidate for such mechanism. Models with large extra dimensions generically contain various objects, such as branes, in the bulk. Collision of the particle with these objects may well produce a BH. An example which allows for a particularly elegant description is collision of the particle with an orbifold fixed point. The latter process can be represented as collision of the particle with its own image(s) under the orbifold symmetry (see figures 1, 4). A BH is expected to be produced once all the images (including the particle itself) are concentrated inside the Schwartzschild radius corresponding to their total energy.

In this scenario the $\mathrm{BH}$ is created far from the SM brane along the extra dimensions. So a natural question is how such a BH can be detected by an observer on the SM brane? We argue that indirect observation of the $\mathrm{BH}$ and its properties, in particular, its Hawking evaporation, is possible if the particle which creates the $\mathrm{BH}$ is charged under the SM gauge group. The latter situation is not unrealistic since the bulk particles in theories with extra dimensions often correspond to the bulk modes of the SM fields. Then, under quite general assumptions described below, the particle flying away from the brane will stretch a string of gauge flux between the SM brane and itself. This string persists when the particle creates $\mathrm{BH}$ and connects the $\mathrm{BH}$ to the SM brane. A brane observer perceives the whole configuration as a charged bound state. Studying the properties of this bound state she can get 
information about the $\mathrm{BH}$. This opens a possibility of (indirect) observation of semiclassical $\mathrm{BHs}$ at the LHC for $M_{*}$ (the quantum gravity scale on the brane) up to a thousand $\mathrm{TeV}$.

The paper is organized as follows. In the next two sections we consider a toy model realizing the scenario outlined above. This enables us to work out the conditions for $\mathrm{BH}$ production and the signatures of this event. Possible generalizations of the model are discussed in section 1 which also contains summary of the results. Some technical details are contained in the appendices.

\section{Toy model}

Let us consider a $d$-dimensional metric, ${ }^{1}$

$$
d s^{2}=A^{2}(y) \eta_{\mu \nu} d x^{\mu} d x^{\nu}-d y^{2}-\gamma_{i j}(\theta) d \theta^{i} d \theta^{j}
$$

where

$$
A(y)=\mathrm{e}^{-k y}
$$

and the coordinates $\theta^{i}, i=1, \ldots, d-5$, describe a $(d-5)$-dimensional compact manifold $\mathcal{M}$ with the metric $\gamma_{i j}$ independent of $y$ and $x^{\mu}$. The size $D_{\mathcal{M}}$ of the manifold $\mathcal{M}$ is assumed to be "large" in the sense of [1], in particular, we consider the case $D_{\mathcal{M}} \gg k^{-1} \gg M_{*}^{-1}$. The space-time is cut off by two codimension-one branes placed at $y=0$ and $y=y_{c}$. The positions of these branes are assumed to be fixed points of $S^{1} / Z_{2}$ orbifold. We do not pretend to describe a realistic configuration by the metric (2.1). Rather, we choose the metric (2.1) for its simplicity allowing us to introduce the main ideas of the paper in a transparent way. In the case $d=5$ (no $\theta$-dimensions) the setup reduces to the well-known Randall-Sundrum model 14]. For $d>5$ and $\mathcal{M}$ a sphere the metric (2.1) is a solution of Einstein equations with a negative cosmological constant and a $(d-6)$-form in the bulk [15].

To localize the SM fields we introduce a 3-brane at $y=\theta_{i}=0$. For simplicity we neglect the back-reaction of this brane on the metric. This brane will be referred to as the "SM brane". ${ }^{2}$ It is straightforward to find the relation between the parameters of the model and the four-dimensional Planck mass determining the strength of gravitational interaction at large distances on the SM brane. Assuming $y_{c} \gtrsim k^{-1}$ one obtains,

$$
M_{\mathrm{Pl}}^{2} \sim M_{*}^{d-2} V_{\mathcal{M}} k^{-1}
$$

where $V_{\mathcal{M}}$ is the volume of the manifold $\mathcal{M}$. By the standard arguments one concludes that $M_{*}$ can be as low as a few $\mathrm{TeV}$ if the product $V_{\mathcal{M}} k^{-1}$ is large enough.

Apart from the SM matter localized on the brane, models with large extra dimensions generically contain matter propagating in the bulk. For example, presence of bulk matter is unavoidable in any field-theoretical realization of the localization mechanism: it

\footnotetext{
${ }^{1}$ Our convention for the signature of the metric is $(+,-,-, \ldots)$.

${ }^{2}$ It is worth stressing the difference between our approach and that of [14]. In our setup the SM brane is placed at the maximum of the warp factor $A$. This is reminiscent of the second Randall-Sundrum model [16]. The hierarchy between $M_{*}$ and the 4-dimensional Planck scale $M_{\mathrm{Pl}}$ is achieved due to the large size of the $\theta$-dimensions, see eq. (2.2)
} 


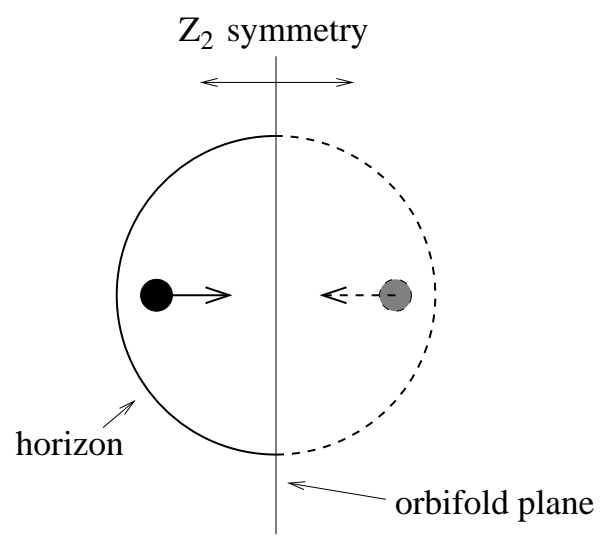

Figure 1: Collision of the particle with an orbifold plane viewed as collision of the particle with its image under $Z_{2}$ symmetry. The black hole horizon is shown schematically.

corresponds to the bulk modes of the fields whose localized excitations describe the SM particles. The rate of production of the bulk modes in collisions of SM particles is model dependent. Typically, it becomes significant above a certain threshold energy $E_{\text {th }}$. The existing experiments set the bound $E_{\text {th }} \gtrsim 1 \mathrm{TeV}$.

Consider a bulk particle produced on the SM brane with energy $E_{0} \gtrsim E_{\text {th }}$. To make the argument as clear as possible let us assume for the moment that the particle moves along the $y$-direction. The time independence of the metric (2.1) implies conservation of the "coordinate" energy $\mathcal{E}$. The latter is related to the physical energy $E$ of the particle in the local inertial frame as follows,

$$
\mathcal{E}=A(y) E
$$

Thus, the particle reaches the plane $y=y_{c}$ with the physical energy $E_{c}=E_{0} / A_{c}$, where $A_{c} \equiv A\left(y_{c}\right)$. Here we arrive to an important point in our discussion. The requirement of $Z_{2}$ symmetry of the metric across the plane $y=y_{c}$ implies that the gravitational field of a particle approaching this plane is equal to the gravitational field of the pair consisting of the particle and its image under $Z_{2}$ reflection, see figure 1. In other words, collision of the particle with the orbifold plane is equivalent to the head-on collision of the particle with its own image. If $E_{c}>M_{*}$ this collision results in $\mathrm{BH}$ formation [17, 18]. The mass of the $\mathrm{BH}$ is estimated as

$$
M_{\mathrm{bh}} \sim E_{c}=E_{0} / A_{c} .
$$

The semiclassical description is valid when $M_{\mathrm{bh}} \gg M_{*}$.

In a curved background, such as ours, the properties of a $\mathrm{BH}$ are different in the cases when its size is smaller or larger than the curvature radius of the background. Small BHs are not affected by the background curvature and are essentially the same as BHs in flat space-time. Their horizon radius is given in $d$ space-time dimensions by the formula

$$
R_{h} \sim\left(M_{\mathrm{bh}} / M_{*}^{(d-2)}\right)^{1 /(d-3)} .
$$


On the other hand, large BHs with $R_{h}>k^{-1}$ are sensitive to the characteristics of the background. An exact solution describing a large $\mathrm{BH}$ in the background (2.1) is unknown; it is not even clear if a stable solution exists at all. The analysis of the Randall-Sundrum case (no large $\theta$-dimensions) performed in [19] shows that the form of the solution, if any, crucially depends on the properties of the mechanism stabilizing the interbrane distance. To avoid these complications we consider only small BHs with $R_{h} \lesssim k^{-1}$ in what follows. This constrains the allowed BH mass from above,

$$
M_{\mathrm{bh}} \lesssim M_{*}\left(M_{*} / k\right)^{d-3}
$$

As $k$ is much smaller than $M_{*}$ this requirement is not very restrictive and leaves a wide parameter region for $\mathrm{BH}$ production.

The above discussion is easily generalized to the case when the particle traveling in the bulk has non-zero momentum along dimensions other than the $y$-direction. All the formulae remain valid with the substitution of the total energy $E$ by the energy of the motion in the direction perpendicular to the orbifold plane,

$$
E_{\perp}=\sqrt{E^{2}-p_{\|}^{2}}
$$

where $p_{\|}$is the component of the particle momentum along the SM brane. Note that the motion of the particle along the $\theta$-directions can be neglected as the corresponding components of the particle momentum are not blue-shifted and hence get small compared to the total energy by the moment the particle reaches the orbifold plane. Evidently, the $\mathrm{BH}$ produced in this general case is not at rest: it slides along the orbifold plane.

Thus we have identified an efficient mechanism of $\mathrm{BH}$ production. Particles leaving the SM brane get accelerated in the bulk and create BHs in collisions with the distant orbifold plane. Note that this process is possible for any value of $M_{*}$ provided the orbifold plane is far enough. We now turn to the question under what conditions creation of $\mathrm{BH}$ can be detected by an observer on the SM brane. The BH emits Hawking radiation with temperature

$$
T_{H} \sim\left(M_{*}^{d-2} / M_{\mathrm{bh}}\right)^{1 /(d-3)} .
$$

The temperature gradually increases as the $\mathrm{BH}$ evaporates. The problem is that the radiation proceeds into the bulk modes which are hard to detect on the brane. Indeed, the temperature $T_{0}$ of the radiation when it reaches the brane is red-shifted compared to (2.4),

$$
T_{0}=T_{H} A_{c}
$$

The bulk modes interact appreciably with the SM matter only if their energy exceeds the threshold for brane-bulk interaction, $T_{0} \geq E_{\mathrm{th}}$. Thus, the radiation of the $\mathrm{BH}$ becomes visible on the brane only during the late stages of $\mathrm{BH}$ evaporation corresponding to $T_{H} \geq E_{\text {th }} A_{c}^{-1}$. This temperature should be still smaller than the quantum gravity scale, $T_{H}<M_{*}$. Combined with the condition for the $\mathrm{BH}$ production this implies the following inequalities,

$$
E_{\mathrm{th}} A_{c}^{-1}<M_{*}<E_{0} A_{c}^{-1} .
$$




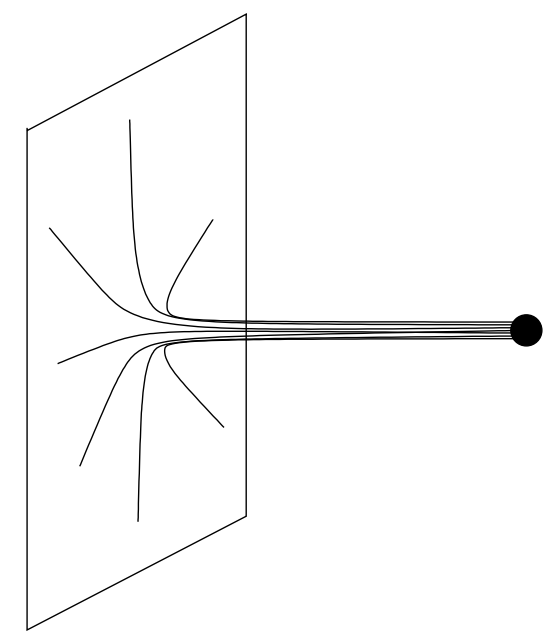

Figure 2: Charged particle placed into the bulk is connected to the brane by a string of gauge flux. The flux opens onto the brane as the field of a point charge.

In principle, the requirement (2.6) leaves non-empty range of parameters. However, in practice $E_{0}$ cannot be much larger than $E_{\mathrm{th}}$. This restricts the masses of the produced BHs to be near the quantum gravity scale $M_{*}$ and thus brings back the theoretical uncertainties discussed in the Introduction. These preliminary considerations show that this scenario of $\mathrm{BH}$ detection, though not completely ruled out, is quite problematic. Note that in the case of more than one large codimension $(d>5)$ the scenario suffers from additional complications due to the dilution of $\mathrm{BH}$ signal in large dimensions transverse to the SM brane. In what follows we concentrate on another option.

\section{String of gauge flux}

A theory with large extra dimensions implies a mechanism of localization of the SM gauge fields on the brane. One generic mechanism [20] (see also [21]) assumes that the gauge theory is in the confining phase in the bulk and thus possesses a mass gap $\mu$. On the brane confinement switches off and the gauge fields become massless. They cannot fly away from the brane if their energy is smaller than $\mu$, i.e. they are localized. The experimental limit on the scale of the bulk confinement is $\mu \gtrsim 1 \mathrm{TeV}$. Assume now that there exist bulk particles charged under the SM gauge group. This is true, for example, if these particle are the bulk modes of the SM fields. When such a particle leaves the SM brane after being created in a high energy collision its gauge flux concentrates into a string stretching between the particle and the brane, see figure 2. This string ensures conservation of gauge charges from the point of view of the observer on the brane who perceives the end of the string as a point charge.

Let us make a digression and discuss the following issue. An explicit realization of the mechanism of ref. [20] for non-Abelian fields in more than four space-time dimensions is unknown. However, the inevitability of the flux-tubes (open strings) attached to the brane goes beyond the specifics of the mechanism of ref. 20]. It follows from just two 
assumptions: dynamical localization of a massless gauge field on the brane and existence of a mass gap in gauge theory in the bulk. Indeed, one can imagine performing a thought experiment in which one removes the test charge away from the brane. Because of the conservation of the gauge charge on the brane, guaranteed by the existence of the zero mode photon and the bulk mass gap, an observer on the brane should measure the same gauge flux through any two-sphere on the brane that surrounds the point from which the charge was removed to the bulk. Because the flux lines cannot break, the only option is that the removed charge continues to produce exactly the same amount of the gauge flux in form of a tube that terminates on the brane. ${ }^{3}$ It is worth emphasizing the importance of the bulk mass gap in the above reasoning: in the absence of the mass gap the situation is more involved 22].

We now return to the consequences of the existence of gauge flux strings for our $\mathrm{BH}$ production scenario. Let the particle which produces the BH carry some of the SM gauge charges. The same charges will be carried by the resulting $\mathrm{BH}$, and therefore the latter will be connected to the SM brane by the string. Probing the end of the string on the SM brane one may extract information about the $\mathrm{BH}$. We proceed to a more careful analysis of this possibility.

Let us specify the acceptable range of parameters. First, we consider the case

$$
\mu>k
$$

so that the effects of the space-time curvature on the inner structure of the string can be neglected. Second, one has to check that the confining force of the string does not prevent the energetic particle from leaving the SM brane and producing a $\mathrm{BH}$. To this end let us calculate the conserved energy of the string stretching between the SM brane and the orbifold plane at $y=y_{c}$. Taking the string tension to be equal to $\mu^{2}$ one obtains

$$
\mathcal{E}_{\text {str }}=\int_{0}^{y_{c}} \mu^{2} A(y) d y=\frac{\mu^{2}}{k}\left(1-\mathrm{e}^{-k y_{c}}\right) .
$$

We see that the energy of the string, as seen by the SM observer, is effectively concentrated in the piece of length $k^{-1}$ near the brane and approaches the asymptotic value $\mu^{2} / k$ at $y_{c} \gg k^{-1}$. The gravitational force pulling the particle into the bulk overcomes the confining force of the string if the energy of the particle is large enough,

$$
E_{0}>\mu^{2} / k \text {. }
$$

As a side remark let us note that particles with energies less than $\mu^{2} / k$ are confined to the SM brane in spite of the gravitational pull. Therefore the gauge field localization mechanism considered here provides also localization of light charged fields.

One more restriction on parameters comes from the requirement that the string should be stable on the time-scales involved in the problem. The physical mechanism which

\footnotetext{
${ }^{3}$ Note that universality of the flux tubes is, in particular, supported by the properties of D-branes in string theory where the presence of massless gauge fields on the brane is related to existence of open strings attached to it.
} 

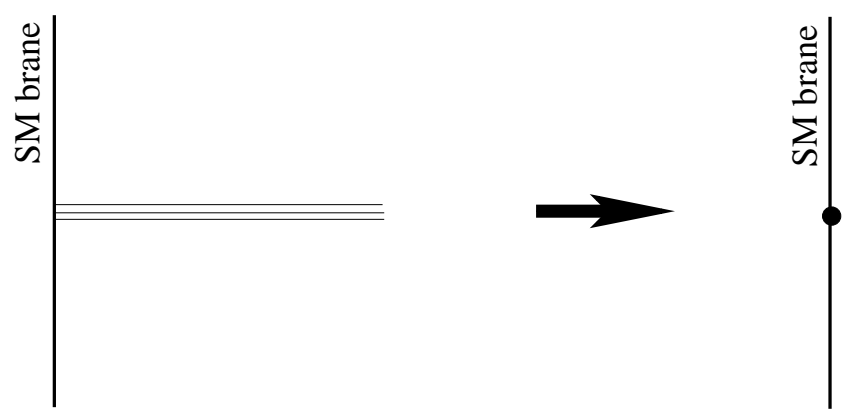

Figure 3: The string can break off the brane by producing one heavy bulk particle and one light particle left on the brane.

destabilizes the string is pair production of charged particles analogous to the Schwinger process. As shown in appendix A, the fastest instability corresponds to the string breaking off the brane by production of one bulk particle and one light SM particle, see figure 3. If the mass $m_{\mathrm{ch}}$ of the lightest charged particle in the bulk is larger than $\mu$ this process is exponentially suppressed. The width of this process is estimated in appendix $\mathrm{A}$,

$$
\Gamma_{\mathrm{str}} \sim \mu \exp \left[-\frac{2 m_{\mathrm{ch}}^{2}}{\mu^{2}} f\left(\frac{m_{\mathrm{ch}} k}{\mu^{2}}\right)\right],
$$

where the function $f(x)$ has the form,

$$
f(x)=\frac{\pi-2 x+2 \sqrt{x^{2}-1} \ln \left(x+\sqrt{x^{2}-1}\right)}{2 x^{2}} .
$$

Below we will see that the string instability can be slow enough to allow detection of BHs.

Now we are ready to work out the signal produced on the SM brane by the BH attached to the brane by the string. At the moment of production the BH-string configuration looks for an observer on the brane as a bound state with the mass ${ }^{4} E_{0}$. The bound state consists of a charged particle (corresponding to the string in the higher-dimensional language) with mass $\mu^{2} / k$ and a neutral component (corresponding to $\mathrm{BH}$ ). As the $\mathrm{BH}$ evaporates the mass of the neutral component, and hence of the whole bound state, decreases. This process continues until the Hawking temperature of the $\mathrm{BH}$ reaches the value $m_{\mathrm{ch}}$. Using the expression (2.4) for the $\mathrm{BH}$ temperature one obtains that this corresponds to the $\mathrm{BH}$ mass

$$
M_{\mathrm{bh}}^{(1)} \sim M_{*}\left(M_{*} / m_{\mathrm{ch}}\right)^{d-3} .
$$

The mass of the bound state observed on the SM brane at this moment is

$$
E_{1} \approx \frac{\mu^{2}}{k}+A_{c} M_{*}\left(\frac{M_{*}}{m_{\mathrm{ch}}}\right)^{d-3} .
$$

At $T_{H}>m_{\mathrm{ch}}$ it becomes possible for the $\mathrm{BH}$ to separate from the string by emitting a charged bulk particle. After that the $\mathrm{BH}$ and the string exist independently of each other:

\footnotetext{
${ }^{4}$ For the sake of the argument we assume that the configuration is produced almost at rest and neglect energy loss in the process of $\mathrm{BH}$ formation.
} 
the $\mathrm{BH}$ has slipped off the hook. For the brane observer this corresponds to the splitting of the bound state into charged and neutral pieces. Only the charged component with the $\operatorname{mass}^{5} \mathcal{E}_{\text {str }}$, see eq. (3.2), continues to be visible to the brane observer.

Let us estimate the lifetime $\Delta t$ of the $\mathrm{BH}$-string bound state. One writes down

$$
\frac{d M_{\mathrm{bh}}}{d t_{c}} \sim-T_{H}^{2},
$$

where $t_{c}=A_{c} t$ is the physical time at the $\mathrm{BH}$ position. Using eq. (2.4) and integrating over the $\mathrm{BH}$ masses from the value at production, $M_{\mathrm{bh}}^{(0)} \approx E_{0} / A_{c}$, to the value (3.6) at the break-off of the bound state one obtains

$$
\Delta t \approx \frac{1}{A_{c} M_{*}}\left[\left(\frac{M_{\mathrm{bh}}^{(0)}}{M_{*}}\right)^{\frac{d-1}{d-3}}-\left(\frac{M_{*}}{m_{\mathrm{ch}}}\right)^{d-1}\right] .
$$

Clearly, positivity of $\Delta t$ requires $M_{\mathrm{bh}}^{(0)}$ to be larger than $M_{\mathrm{bh}}^{(1)}$, eq. (3.6). The lifetime $\Delta t$ of the bound state can be very large, see eq. (3.12) below. It makes the signature of $\mathrm{BH}$ production very distinctive: formation of a heavy charged long-lived bound state with the mass slowly changing in time.

Further information can be obtained by monitoring the charged component left after the splitting of the BH-string bound state. The behavior of this charged component depends on the ratio between $m_{\mathrm{ch}}$ and $\mu^{2} / k$. If $m_{\mathrm{ch}}>\mu^{2} / k$ the gravitational pull forces the bulk particle terminating the string to remain on the orbifold plane. The string remains stretched between the orbifold plane and the SM brane until it breaks away from the brane after time $\Gamma_{\text {str }}^{-1}$. What is seen by the brane observer is a heavy charged particle with mass $\mathcal{E}_{\text {str }}$ decaying with lifetime $\Gamma_{\text {str }}^{-1}$ into a SM particle with the same charges plus something invisible. Conversely, if $m_{\mathrm{ch}}<\mu^{2} / k$ the string collapses onto the SM brane immediately after the separation from the $\mathrm{BH}$ and releases its energy decaying into the SM fields.

The above discussion is valid if the lifetime of the BH-string bound state is smaller than the decay time of the string,

$$
\Delta t<\Gamma_{\mathrm{str}}^{-1} .
$$

Another requirement is that the BH-string system must be bound strongly enough to be considered as a single object. This amounts to requiring that the frequencies of internal oscillations of the bound state are large compared to its inverse lifetime. In appendix B we find that the spectrum of the internal oscillations of the BH-string system consists of of a low lying mode with frequency

$$
\omega_{0}=A_{c}^{3 / 2} k \sqrt{3\left(1+\frac{\mu^{2}}{A_{c} k M_{\mathrm{bh}}}\right)}
$$

and a tower of modes with frequencies,

$$
\omega_{n} \approx \pi A_{c} k(n+1 / 2), \quad n=1,2, \ldots .
$$

\footnotetext{
${ }^{5}$ The small contribution $A_{c} m_{\mathrm{ch}}$ to the total mass of the charged component, coming from the particle terminating the string on the orbifold plane, can be neglected.
} 
Note that the lowest frequency (3.8) is sensitive to the $\mathrm{BH}$ mass: it grows as the $\mathrm{BH}$ evaporates. On the other hand the higher eigenfrequencies (3.9) correspond to the intrinsic oscillations of the string: they do not depend on the mass of the $\mathrm{BH}$ and are present even after the string separates from the $\mathrm{BH}$. The $\mathrm{BH}$-string system is strongly bound if

$$
\omega_{0} \gg \Delta t^{-1}
$$

The quantities entering into eqs. (3.7), (3.10) have complicated dependence on the parameters of the model. A careful study is needed to work out the region of parameter space where these requirement as well as other constraints (2.3), (3.1), (3.3) are satisfied. Such a study is beyond the scope of the present article. We limit ourselves to rough estimates which show that the allowed region is likely to contain phenomenologically interesting values. As an example we consider

$$
\begin{aligned}
& d=7, \quad M_{*}=1000 \mathrm{TeV}, \quad A_{c}=10^{-12}, \\
& k=0.2 \mathrm{TeV}, \quad \mu=1 \mathrm{TeV}, \quad E_{0}=m_{\mathrm{ch}}=8 \mathrm{TeV} .
\end{aligned}
$$

Using the equations given above one obtains,

$$
\begin{array}{rlrl}
M_{\mathrm{bh}}^{(0)} & =3 \cdot 10^{15} \mathrm{GeV}, & & M_{\mathrm{bh}}^{(1)}=2.4 \cdot 10^{14} \mathrm{GeV} \\
E_{1} & =5.24 \mathrm{TeV}, & \mathcal{E}_{\mathrm{str}}=5 \mathrm{TeV} \\
\Delta t & \approx 1.6 \cdot 10^{20} \mathrm{GeV}^{-1} \approx 10^{-4} \mathrm{~s}, \\
\Gamma_{\mathrm{str}}^{-1} & \sim 0.6 \cdot 10^{25} \mathrm{GeV}^{-1} \approx 4 \mathrm{~s} \\
\omega_{0} & =(5.7 \div 16) \cdot 10^{-16} \mathrm{GeV} \approx(0.8 \div 2.4) \cdot 10^{9} \mathrm{~s}^{-1}, &
\end{array}
$$

where in the last line we have indicated the range of variation of $\omega_{0}$ due to the change in the $\mathrm{BH}$ mass. It is straightforward to check that these values indeed satisfy all the constraints. Note that the lifetime of the BH-string bound state and the decay time of the string are very large by particle physics standards.

Let us conclude the analysis of our toy model by indicating a few more possible signatures of the $\mathrm{BH}$-string bound system. As mentioned above the lowest frequency of this system depends on time due to the change in the BH mass. A measurement of this dependence would give an accurate information on the $\mathrm{BH}$ evaporation process. Such a measurement could be particularly useful in the case of a very slow BH evaporation implying a long lifetime of the BH-string bound state. We have nothing to say about the experimental feasibility of such a measurement.

Finally, it is known [23] that a string embedded into a $\mathrm{BH}$ provides an additional channel for the Hawking radiation. This effect gives rise to thermal oscillations of the string in the BH-string system. The corresponding temperature $T_{0}$, as seen from the SM brane, is given by eq. (2.5). The typical value of $T_{0}$ is quite small compared to the mass of the constituents of the BH-string system. For example, the choice (3.11) gives $T_{0} \sim 1 \mathrm{eV}$. It is not clear if these oscillations can lead to observable effects on the brane. We leave this issue for future studies. 


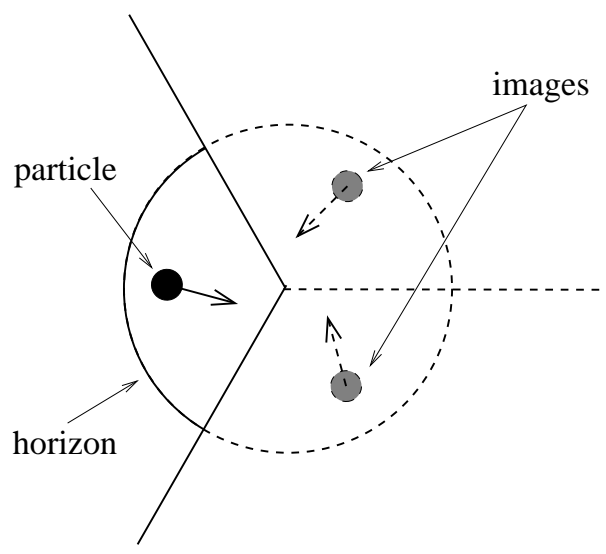

Figure 4: Collision of particle with an $\mathbb{R}^{2} / Z_{3}$ orbifold fixed manifold is equivalent to collision with two images. The collision produces a black hole.

\section{Generalizations and summary}

The toy model studied above admits various generalizations. With slight changes of the formulae of the previous sections the scenario is applicable to a wide class of higherdimensional backgrounds. One class of generalizations concern the mechanism of $\mathrm{BH}$ production. Instead of considering collision of a particle with an orbifold plane one may consider collision with an orbifold fixed manifold of codimension higher than one. Such a collision also results in $\mathrm{BH}$ formation, see figure 4 where a collision of the particle with an "orbifold string" is shown as an example. More generally, BHs may result from particle collisions with other branes present in the bulk.

Other generalizations correspond to the case when the parameters governing the strength of gravitational and gauge interactions vary across the bulk. For example, gravity can be coupled to a dilaton field so that the higher-dimensional gravitational action takes the form,

$$
S_{g}=-M_{*}^{d-2} \int d^{d} x \sqrt{|g|} R \mathrm{e}^{-\phi} .
$$

In this case the strength of gravitational interaction depends on the position in the extra dimensions even from the point of view of bulk observers: if $\phi$ has a non-trivial profile in the extra dimensions the local Planck scale $M_{*} \mathrm{e}^{-\phi /(d-2)}$ varies accordingly. In particular, gravity may become stronger deep in the bulk than near the SM brane. Yet another possibility is that the gravitational interaction on the brane is suppressed compared to the bulk by the presence of a brane Einstein-Hilbert term in the gravitational action [8, 24]. All these effects, together with possible dependence of the confinement scale $\mu$, the mass of the charged particle $m_{\mathrm{ch}}$ and the background curvature scale $k$ on the position in the bulk, can affect the properties (mass, lifetime, etc.) of the string and of the BH-string bound state.

To sum up, we have proposed a generic scenario of production and detection of semiclassical BHs in the framework of theories with large extra dimensions. In contrast to the standard picture of $\mathrm{BH}$ production in particle collisions the $\mathrm{BHs}$ in our scenario are 
created away from the brane in the regions of the bulk where gravity is stronger than on the brane. The signatures of the scenario are largely different from those usually studied in the context of $\mathrm{BH}$ production.

The details of a given realization of this scenario are model dependent. However, the main ideas are summarized as follows. A collision on the SM brane produces a particle which can freely propagate in the bulk. This particle flies away from the brane into the region where its energy is much higher than the local Planck scale. There it produces a BHs in collisions with some object (orbifold fixed manifold, brane, etc.) in the bulk. If the bulk particle is charged under the SM gauge group, the resulting BH is connected to the SM brane by a string of gauge flux. From the point of view of the SM observer the system behaves as a heavy bound state with charged and neutral components. The mass of the latter component slowly decreases with time due to evaporation of the $\mathrm{BH}$. After some, generically large, time the $\mathrm{BH}$ separates from the string which corresponds to the break off of the bound state. This behavior provides a distinctive signature of our scenario.

Other possible signatures depending of the details of a given realization include observation of low-frequency excitations of the bound state, thermal oscillations of the system due to BH Hawking radiation, or decay of the charged component after the break off of the bound state. It would be interesting to study the signatures of the scenario proposed in this paper in more detail and construct viable extra-dimensional models where this scenario is realized.

\section{Acknowledgments}

We thank D. Gorbunov and M. Redi for useful discussions. We are grateful to O. Pujolas and I. Tkachev for encouraging interest. This work was supported in part by the David and Lucile Packard Foundation Fellowship for Science and Engineering, by NSF grant PHY-0245068 and by EU 6th Framework Marie Curie Research and Training network "UniverseNet" (MRTN-CT-2006-035863).

\section{A. Stability of the string in warped background}

In this appendix we study the process of string breaking by pair production of charged particles in the warped background (2.1). We consider a string stretched between the SM brane at $y=0, \theta_{i}=0$ and the point $y=y_{c}, \theta_{i}=0$ on the orbifold plane. If the mass $m_{\mathrm{ch}}$ of the charged particles in the bulk is larger than the scale $\mu$ of the string tension, the pair production is a tunneling process and can be analyzed by semiclassical methods.

The case of an infinite string in flat space-time was studied in [25] where the probability of string decay per unit time per unit length was found,

$$
P=\mu^{2} A\left(\frac{m_{\mathrm{ch}}}{\mu}\right) \exp \left[-\frac{\pi m_{\mathrm{ch}}^{2}}{\mu^{2}}\right],
$$

where $A(x)$ is some function whose form is not important as long as the exponential factor is small. We will omit this function in what follows. The result (A.1) is also applicable in 
curved backgrounds if the distance $R=m_{\mathrm{ch}} / \mu^{2}$ between the particles at the moment of production is small compared to the curvature radius of the background. In our case this corresponds to $m_{\mathrm{ch}} k / \mu^{2} \ll 1$. Integrating (A.1) over the length of the string and taking into account the dilation of the time intervals due to warping we obtain the width of the string decay via pair production in the bulk for the case $m_{\mathrm{ch}} k / \mu^{2} \ll 1$,

$$
\Gamma_{\text {bulk }}=\int P A(y) d y \sim \frac{\mu^{2}}{k} \exp \left[-\frac{\pi m_{\mathrm{ch}}^{2}}{\mu^{2}}\right] .
$$

On the other hand, the pair production in the bulk is completely suppressed by gravitational effects if $m_{\mathrm{ch}} k / \mu^{2}>1$. Indeed, consider the change in the conserved energy of the system when the string breaks into two parts terminated by charged particles at the positions $y=y_{1}, y=y_{2}$. One obtains,

$$
\Delta \mathcal{E}=\left(m_{\text {ch }}-\frac{\mu^{2}}{k}\right) \mathrm{e}^{-k y_{1}}+\left(m_{\text {ch }}+\frac{\mu^{2}}{k}\right) \mathrm{e}^{-k y_{2}} .
$$

We see that if $m_{\mathrm{ch}} k / \mu^{2}>1$ the change of energy is positive and thus the pair production in the bulk is impossible.

In our case there is one more channel of the string instability compared to the case of the infinite string. Namely, the string can break away from the brane via pair production of a bulk particle and a SM particle, see figure 3. As this process requires production of only one heavy bulk particle one may expect that it is preferable to the bulk pair production considered above. We are going to see that this is indeed the case.

On general grounds the width of the process depicted in figure 3 has the form

$$
\Gamma=\mu B\left(m_{\mathrm{ch}} / \mu, k / \mu\right) \exp \left[-S_{B}\right],
$$

where $S_{B}$ is the action of the bounce solution describing the string decay, and $B$ is some function which we will omit in what follows. The bounce solution extremizes the Euclidean action of the system consisting of the charged particle attached to the end of the string,

$$
S_{E}=\mu^{2} \int \sqrt{\operatorname{det} \bar{g}_{a b}} d^{2} \xi+m_{\mathrm{ch}} \int d s,
$$

where $\bar{g}_{a b}$ is the induced metric on the string worldsheet parameterized by the coordinates $\xi^{a}, a=1,2$; in the second term integration is performed over the trajectory of the end of the string. In writing down the action (A.4) we have neglected the contribution of the light SM particle living on the brane. The bounce solution has the form of a hole in the string worldsheet bounded on one side by the trajectory of the bulk particle and on the other side by the brane, see figure 5. It is convenient to bring the background metric into the conformally flat form by introducing the coordinate

$$
\zeta=k^{-1} \mathrm{e}^{k y}
$$

and parameterize the particle trajectory by $\zeta\left(t_{E}\right)$, where $t_{E}$ is the Euclidean time. Then the bounce action takes the following form,

$$
S_{B}=\int d t_{E}\left[-\frac{\mu^{2}}{k}+\frac{\mu^{2}}{k^{2} \zeta}+\frac{m_{\mathrm{ch}}}{k \zeta} \sqrt{1+\left(\frac{d \zeta}{d t_{E}}\right)^{2}}\right]
$$




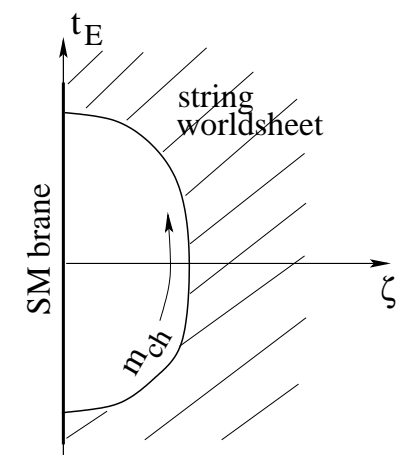

Figure 5: The Euclidean solution describing the string breaking away from the brane.

where we subtracted the action of the "vacuum" configuration corresponding to the string without holes. Extremization of the action (A.6) over the trajectories of the particle yields

$$
S_{B}=\frac{2 m_{\mathrm{ch}}^{2}}{\mu^{2}} \int_{0}^{1} d z \frac{\sqrt{1-z^{2}}}{1+\frac{m_{\mathrm{ch}} k}{\mu^{2}} z}=\frac{2 m_{\mathrm{ch}}^{2}}{\mu^{2}} f\left(\frac{m_{\mathrm{ch}} k}{\mu^{2}}\right),
$$

where the function $f$ is defined in eq. (3.5). Substituting this expression into eq. (A.3) one obtains the formula (3.4) of the main text. Note that at $k=0$ equation (A.7) gives $S_{B}=\pi m_{\mathrm{ch}}^{2} / 2 \mu^{2}$ which is half of the suppression exponent in eq. (A.2). Thus the width of the process of figure 3 is much larger than the width (A.2) of pair production in the bulk.

\section{B. Internal oscillations of BH-string bound system}

Let us study the spectrum of excitations of the BH-string bound system. Of main interest to us are oscillations of the string in the directions parallel to the SM brane: these oscillations correspond to the motion of the charged component of the bound state visible by the brane observer. In what follows we concentrate on this type of excitations and leave aside possible oscillations of the string in the $\theta$-directions.

The worldsheet of the string is described by the functions $x^{\alpha}(t, \zeta)$, where $x^{\alpha}, \alpha=1,2,3$ are the spatial coordinates parallel to the brane and $\zeta$ is the coordinate along the fifth dimension defined in (A.5). To simplify notations we omit the index $\alpha$ below. The string is stretched between the SM brane at $\zeta=k^{-1}$ and the $\mathrm{BH}$ sitting at $\zeta_{c}=k^{-1} / A_{c}$. For our present purposes the $\mathrm{BH}$ can be treated as a point mass $M_{\mathrm{bh}}$ moving along the $x$-direction, $x_{\mathrm{bh}}(t)=x\left(t, \zeta_{c}\right)$. Expanding the Nambu-Goto action of the string and the action of the point mass around the static configuration $x(t, \zeta)=0$ one writes down the action for the small oscillations of the BH-string system,

$$
S=\frac{\mu^{2}}{2} \int \frac{d t d \zeta}{(k \zeta)^{2}}\left[\left(\partial_{t} x\right)^{2}-\left(\partial_{\zeta} x\right)^{2}\right]+\left.\frac{M_{\mathrm{bh}}}{2} \int \frac{d t}{k \zeta_{c}}\left(\partial_{t} x\right)^{2}\right|_{\zeta=\zeta_{c}} .
$$

From this action one derives the equation for the string oscillations,

$$
-\frac{\partial^{2} x}{\partial t^{2}}+\frac{\partial^{2} x}{\partial \zeta^{2}}-\frac{2}{\zeta} \frac{\partial x}{\partial \zeta}=0
$$


supplemented by the boundary conditions

$$
\begin{aligned}
\left.\partial_{\zeta} x\right|_{\zeta=k^{-1}} & =0 ;, \\
\mu^{2} \partial_{\zeta} x+\left.M_{\mathrm{bh}}\left(k \zeta_{c}\right) \partial_{t}^{2} x\right|_{\zeta=\zeta_{c}} & =0 .
\end{aligned}
$$

The solutions of eqs. (B.1), (B.2) have the form

$$
x_{\omega}=C_{\omega}\left[(1+i \omega \zeta) \mathrm{e}^{-i \omega\left(\zeta-k^{-1}\right)}-(1-i \omega \zeta) \mathrm{e}^{i \omega\left(\zeta-k^{-1}\right)}\right] \mathrm{e}^{-i \omega t},
$$

where $C_{\omega}$ is an arbitrary constant and the eigenfrequencies $\omega$ satisfy the following equation,

$$
\frac{\operatorname{tg}\left[\omega\left(\zeta_{c}-k^{-1}\right)\right]}{\omega \zeta_{c}}=\left(1-\frac{\mu^{2}}{k M_{\mathrm{bh}}}\right)^{-1} .
$$

For $M_{\mathrm{bh}} \gg \mu^{2} / k$ this equation leads to the expressions (3.8), (3.9).

\section{References}

[1] N. Arkani-Hamed, S. Dimopoulos and G.R. Dvali, The hierarchy problem and new dimensions at a millimeter, Phys. Lett. B 429 (1998) 263 hep-ph/9803315;

I. Antoniadis, N. Arkani-Hamed, S. Dimopoulos and G.R. Dvali, New dimensions at a millimeter to a Fermi and superstrings at a TeV, Phys. Lett. B 436 (1998) 257 hep-ph/9804398;

N. Arkani-Hamed, S. Dimopoulos and G.R. Dvali, Phenomenology, astrophysics and cosmology of theories with sub-millimeter dimensions and TeV scale quantum gravity, Phys. Rev. D 59 (1999) 086004 hep-ph/9807344.

[2] G. Dvali, Black holes and large- $N$ species solution to the hierarchy problem, arXiv:0706.2050.

[3] G. Dvali and M. Redi, Black hole bound on the number of species and quantum gravity at $L H C$, arXiv:0710.4344.

[4] J.D. Bekenstein, Black holes and entropy, Phys. Rev. D 7 (1973) 2333.

[5] S.W. Hawking, Particle creation by black holes, Commun. Math. Phys. 43 (1975) 199 [Erratum ibid. 46 (1976) 206].

[6] T. Banks and W. Fischler, A model for high energy scattering in quantum gravity, hep-th/9906038.

[7] R. Emparan, G.T. Horowitz and R.C. Myers, Black holes radiate mainly on the brane, Phys. Rev. Lett. 85 (2000) 499 hep-th/0003118.

[8] G.R. Dvali, G. Gabadadze, M. Kolanovic and F. Nitti, Scales of gravity, Phys. Rev. D 65 (2002) 024031 hep-th/0106058.

[9] S.B. Giddings and S.D. Thomas, High energy colliders as black hole factories: the end of short distance physics, Phys. Rev. D 65 (2002) 056010 hep-ph/0106219].

[10] S. Dimopoulos and G.L. Landsberg, Black holes at the LHC, Phys. Rev. Lett. 87 (2001) 161602 hep-ph/0106295. 
[11] S.B. Giddings, High-energy black hole production, AIP Conf. Proc. 957 (2007) 69 arXiv:0709.1107.

[12] P. Meade and L. Randall, Black holes and quantum gravity at the LHC, arXiv:0708.3017.

[13] H. Yoshino and R.B. Mann, Black hole formation in the head-on collision of ultrarelativistic charges, Phys. Rev. D 74 (2006) 044003 gr-qc/0605131.

[14] L. Randall and R. Sundrum, A large mass hierarchy from a small extra dimension, Phys. Rev. Lett. 83 (1999) 3370 hep-ph/9905221.

[15] T. Gherghetta, E. Roessl and M.E. Shaposhnikov, Living inside a hedgehog: higher-dimensional solutions that localize gravity, Phys. Lett. B 491 (2000) 353 hep-th/0006251.

[16] L. Randall and R. Sundrum, An alternative to compactification, Phys. Rev. Lett. 83 (1999) 4690 hep-th/9906064.

[17] P.D. D'Eath and P.N. Payne, Gravitational radiation in high speed black hole collisions. 1. Perturbation treatment of the axisymmetric speed of light collision, Phys. Rev. D 46 (1992) 658; Gravitational radiation in high speed black hole collisions. 2. Reduction to two independent variables and calculation of the second order news function, Phys. Rev. D 46 (1992) 675; Gravitational radiation in high speed black hole collisions. 3. Results and conclusions, Phys. Rev. D 46 (1992) 694.

[18] D.M. Eardley and S.B. Giddings, Classical black hole production in high-energy collisions, Phys. Rev. D 66 (2002) 044011 gr-qc/0201034.

[19] S.B. Giddings, High energy QCD scattering, the shape of gravity on an IR brane and the Froissart bound, Phys. Rev. D 67 (2003) 126001 hep-th/0203004.

[20] G.R. Dvali and M.A. Shifman, Domain walls in strongly coupled theories, Phys. Lett. B 396 (1997) 64 [Erratum ibid. B 407 (1997) 452] hep-th/9612128].

[21] S.L. Dubovsky and V.A. Rubakov, On models of gauge field localization on a brane, Int. J. Mod. Phys. A 16 (2001) 4331 hep-th/0105243.

[22] S.L. Dubovsky, V.A. Rubakov and P.G. Tinyakov, Is the electric charge conserved in brane world?, JHEP 08 (2000) 041 hep-ph/0007179.

[23] V.P. Frolov and D. Fursaev, Mining energy from a black hole by strings, Phys. Rev. D 63 (2001) 124010 hep-th/0012260.

[24] G.R. Dvali, G. Gabadadze and M. Porrati, $4 D$ gravity on a brane in $5 D$ Minkowski space, Phys. Lett. B 485 (2000) 208 hep-th/0005016;

G.R. Dvali, G. Gabadadze, M. Kolanovic and F. Nitti, The power of brane-induced gravity, Phys. Rev. D 64 (2001) 084004 hep-ph/0102216.

[25] A. Vilenkin, Cosmological evolution of monopoles connected by strings, Nucl. Phys. B 196 (1982) 240 . 